\title{
LA VIRTUD DE LA PRUDENCIA EN EL PENSAMIENTO DE ALASDAIR MACINTYRE
}

\author{
David Lorenzo Izquierdo \\ Universidad de Barcelona \\ dlorenzo@santjoandedeu.edu.es
}

\begin{abstract}
Resumen / ABSTRACT
El concepto de virtud de Alasdair MacIntyre ha recibido, por parte de la crítica, un tratamiento no sistemático y poco profundo. En concreto, la virtud de la prudencia (la 'phronesis') ha recibido escasa atención. La prudencia es una virtud muy importante en el pensamiento de MacIntyre, aunque el autor no la haya analizado de manera unitaria y sistemática. Sus principales propuestas sobre el ser humano y la investigación moral no pueden ser comprendidas sin la virtud de la prudencia. El objetivo de este artículo es ofrecer una exposición y un análisis de dicha virtud en el pensamiento de MacIntyre.
\end{abstract}

PALABRAS ClAVE: MacIntyre, virtud, prudencia, florecimiento.

\section{THE VIRTUE OF PRUDENCE IN ALASDAIR MACINTYRE'S THINKING}

The concept of virtue by Alasdair MacIntyre has been treated by his critics in a unsystematic and shallow way. In particular, the virtue of prudence (the 'phronesis') has received little attention. Prudence is a very important virtue in MacIntyre's thought, although the author has not analyzed it in a unitary and systematic way. His main proposals about human being and moral enquiry can not be understood without the virtue of prudence. The aim of this paper is to offer an exposition and an analysis of this virtue in MacIntyre's thought.

KEYWORDS: MacIntyre, virtue, prudence, flourishing.

\section{Introducción}

D) Alasdair MacIntyre es un autor con una larga trayectoria intelectual. $\mathrm{Su}$ pensamiento ha discurrido, fundamentalmente, por el marxismo, el aristotelismo y el tomismo ${ }^{1}$. En ella, conceptos como tradición, virtud, comunidad, práctica, etc., han sido fundamentales en la vertebración de sus propuestas $\mathrm{y}$, a la vez, han tenido

1 Una breve descripción de esa trayectoria la hace él mismo en el "Prefacio" del libro The Tasks of Philosophy. Selected essays (vol. 1) (2006). Ver también el "Prefacio" de Ethics in the Conflicts of Modernity (2016). 
gran influencia en la filosofía moral contemporánea y, en cierto modo, han hecho de MacIntyre un pensador relevante.

Se ha escrito mucho sobre dichos conceptos, especialmente sobre el de tradición. El concepto de virtud también ha sido objeto de interés y debate en el ámbito académico, sobre todo a partir de la publicación en 1981 de After Virtue (Tras la virtud), aunque pensamos que su tratamiento ha sido, en comparación con el que han recibido otros conceptos, menos sistemático y profundo.

Un ejemplo de ello es el tratamiento que ha recibido la virtud de la prudencia que MacIntyre propone en sus escritos. La reflexión de nuestro autor sobre dicha virtud ha recibido atención por parte de la crítica. Este hecho es, en nuestra opinión, paradójico a la luz de tres cuestiones importantes: en primer lugar, el hecho de que el concepto mismo de virtud ha sido muy importante en el pensamiento de nuestro autor; en segundo lugar, que la figura de MacIntyre ha supuesto una gran contribución a la recuperación del interés por ese concepto en la filosofía moral contemporánea; y, por último, que la virtud de la prudencia ha sido una virtud destacada en la tradición filosófica occidental (y, en concreto, en dos autores fundamentales para MacIntyre, como son Aristóteles y Tomás de Aquino).

Conviene a la vez señalar que el pensamiento de nuestro autor, en nuestra opinión, no añade nada nuevo en lo esencial a la reflexión sobre la prudencia que ofrecen Aristóteles y Tomás de Aquino. Sin embargo, tiene el valor de recuperar esa virtud e introducirla y adaptarla a estructuras y conceptos contemporáneos, de modo que el concepto adquiere matices nuevos y, a la vez, permite explicar dichas estructuras de una manera más completa e iluminadora. Por ello pensamos que es útil exponer y analizar de manera específica y sistemática la reflexión sobre la virtud de la prudencia que ofrece A. MacIntyre en su propuesta filosófica ${ }^{2}$.

El análisis que se expone aquí del tema pretende ser conceptual, no evolutivo o histórico. Es decir, interesa aquí la visión unitaria sobre dicho concepto que puede extraerse del pensamiento de nuestro autor, no tanto describir su evolución a lo largo de su trayectoria personal e intelectual ${ }^{3}$. Por ello, el análisis y la reflexión que se presentan

2 Este artículo toma algunos textos de un trabajo previo del autor (escrito en 2016 y publicado en portugués en 2018): "O conceito de virtude no pensamento de Alasdair MacIntyre", en Carvalho, Helder B. A. (ed.), Tradição, Moralidade e Racionalidade: Em diálogo com Alasdair MacIntyre, Porto Alegre, Editora Fi and EDUFPI, 2018, pp. 249-267. El autor agradece el permiso de la editorial para aprovechar ese material previo para la elaboración de este trabajo.

3 El concepto de virtud adquiere un papel eminente en el pensamiento de nuestro autor a partir de la publicación de After Virtue en 1981. Por ello este trabajo se basa, salvo en alguna excepción, en las obras publicadas a partir de ese libro. Para elaborar este texto, los siguientes libros publicados tras 1981 han sido consultados aunque su contenido, salvo en el caso de algún tema de detalle, no ha sido utilizado: The Tasks of Philosophy. Selected essays (vol. 1) (2006), Ethics and Politics. Selected essays (vol. 2) (2006), Edith Stein: 
suponen y requieren una re-construcción de su pensamiento, esto es, aunar ideas que el autor ha expuesto en obras y momentos distintos.

Para comprender el papel de la prudencia en el pensamiento de nuestro autor, es necesario explicar el contexto en el que está situada y que le da sentido. Dicho contexto está definido por las prácticas y el florecimiento.

\section{La virtud y la práctica}

El concepto de práctica es un concepto central en el pensamiento macintyreano. MacIntyre define la práctica (practice) como una actividad coherente y cooperativa socialmente establecida, mediante la que se realizan los bienes inherentes a ella intentando, así, lograr los modelos de excelencia apropiados -a ella-, con el resultado de que la capacidad de lograr esa excelencia y los conceptos de los fines y los bienes que conlleva se extienden sistemáticamente. Ejemplos de prácticas son la arquitectura, el fútbol, la agricultura y, en general, artes, ciencias, juegos intelectuales y atléticos (1981, pp. 187-188, 200-201).

Las prácticas configuran la red de espacios o actividades en las que el individuo desarrolla su vida ordinaria: son el espacio natural en el que vive. Un individuo no vive 'en general' sino que vive 'en las prácticas'. Por eso, afirma MacIntyre que esas prácticas definen los papeles (roles) que la persona puede desarrollar en la sociedad, la sitúan ante ella -podríamos decir-. Y sostiene que, en toda sociedad, los individuos, por sus preferencias, situación, grupo, etc., representan o encarnan papeles: dentista, pensionista, sacerdote, etc. (1981, pp. 28-32).

Toda práctica se articula o se vertebra con tres elementos: unos bienes, unos modelos de excelencia -autoridades-y unas reglas que obedecer (1981, pp. 189-190). Veámoslos brevemente.

Los bienes que entran en juego en las prácticas son de dos tipos según MacIntyre: externos e internos. Los bienes externos se pueden conseguir con cualquier práctica y no son compartibles: poder, dinero, rango, éxito, etc. Los bienes internos afectan o se relacionan con la excelencia propia de esa práctica: MacIntyre pone el ejemplo de la pintura retratista, que tiene en el mejor retrato y su técnica adecuada su bien interno. Por ello, estos bienes se pueden conseguir solo con una práctica (o prácticas similares), son solo reconocibles por los que participan en ella y son bienes compartibles, pues su logro es un bien para toda la comunidad. Los bienes internos conforman el 'telos' (la finalidad) de la práctica como actividad. Ese fin es lo que da sentido a las reglas (y a su aplicación) (1981, pp. 188-194; 1990b, p. 26; 1996b, p. 80).

A Philosophical Prologue (2006), God, Philosophy, Universities (2009) y Ethics in the Conflicts of Modernity (2016). 
Las reglas (las normas) son, genéricamente, guías del comportamiento. Toda práctica tiene unas reglas (técnicas y morales) que guían al individuo hacia el bien interno. Por otra parte, entrar en una práctica es entrar en contacto con los que la practican y la han practicado, $\mathrm{y}$, en concreto, con los que mejor la han practicado (los que más se han acercado al 'telos') son los modelos (autoridades). El individuo aprende de ellos y con ellos. Son los modelos los que enseñan la definición y la aplicación de las reglas, que son elementos fundamentales porque guían la actividad del agente para que ésta se ajuste a lo requerido por el 'telos' de esa práctica (1981, pp. 194-195).

A la luz de estas explicaciones, se desprende que la virtud se relaciona con la práctica de dos modos:

$\left.1^{\circ}\right)$ Las virtudes son necesarias para que el individuo pueda reconocer y realizar los bienes internos a las prácticas: sin unas cualidades mínimas, el individuo no puede alcanzar la excelencia propia de la actividad en que se halla (1981, pp. 195-196; 1991b, p. 262).

$2^{\circ}$ ) Las prácticas -su actividad-son de hecho el principal camino de adquisición y crecimiento en virtudes, aunque no es el único (pues también se adquieren en la educación específicamente moral), ni tampoco el único ámbito en que se ejercen (1981, pp. 186-187, 200-201; 1996b, p. 80; 1999a, pp. 88-89).

Ya hemos visto que entre el individuo y el bien interno de la práctica median los modelos y las reglas. Alcanzar el bien interno pasa por ellos, por su aprovechamiento y correcta articulación. Seguir la enseñanza de los modelos o autoridades y aprehender y aplicar las reglas son actos que el individuo puede realizar solo a través de las virtudes. Los modelos enseñan, por una parte, a reconocer, valorar y alcanzar los bienes internos; $\mathrm{y}$, por otra, a definir y aplicar las reglas. Ambos elementos (los bienes y los modelos) impiden cualquier visión o juicio subjetivista (individualista) de la práctica. Sin embargo, para que el individuo sea capaz de asumir todo eso, necesita virtudes (1981, pp. 190-191, 194-195).

A la luz de la consideración de todos sus elementos, MacIntyre sostiene que la práctica es un concepto y una realidad con tal estructura que solo puede ser entendida desde una concepción aristotélica de la acción. Dice en la entrevista "Después de Tras la virtud" que entender y realizar una práctica pasa por entender que tiene un 'telos' y que requiere educación en virtudes para alcanzarlo, esquema o estructura propios del aristotelismo y comprensibles adecuadamente sólo desde él (1990d, p. 89).

\section{La virtud y el florecimiento}

El ámbito de las prácticas supone un primer nivel de crecimiento y ejercicio de virtudes. El siguiente, de naturaleza y alcance más amplio, es el del florecimiento.

Para MacIntyre, el florecimiento consiste en el desarrollo máximo del hombre como ser humano, desarrollo que tiene una vertiente física (biológica) y una vertiente 
intelectual-moral (referida al desarrollo del razonamiento práctico). La identidad humana es corporal y anímica, implica y requiere el desarrollo del cuerpo. No obstante, el florecimiento propiamente humano se distingue por su vertiente intelectual-moral, (1990a, pp. 196-197, 1999a, pp. 8-9).

El concepto 'florecimiento' hace posible considerar la vida individual como una narración, como una 'unidad narrativa' ('narrative unity'), término desarrollado por MacIntyre en After Virtue. Este concepto es quizá uno de los más famosos de su pensamiento. El autor sostiene en After Virtue que la vida individual puede entenderse como una unidad, como un todo con un comienzo, un desarrollo y un final. Puede entenderse así teóricamente porque ya es de hecho entendida así prácticamente, en las acciones particulares y concretas del individuo (1981, pp. 118, 130). Uniendo ambos conceptos ('florecimiento' y 'unidad narrativa'), es posible afirmar que la vida humana - cada vida humana- es precisamente la narración del florecimiento individual.

De esta manera, las acciones individuales pueden considerarse como medios para el florecimiento, proceso que es tanto teórico como práctico. Aunque MacIntyre no habla del término 'eudaimonía', puede verse que el concepto de florecimiento toma o asume la estructura básica de la teoría aristotélica de la felicidad (Mas, 1996, p. 163; Corral, 1997, p. 134). Claro ejemplo de ello es su artículo "Plain Persons and Moral Philosophy: Rules, Virtues and Goods" (de 1992), donde sostiene la tesis de que toda vida individual tiene una estructura proto-aristotélica ("proto-Aristotelian structure") (1992, p. 138).

Hemos comentado antes que el individuo vive 'en las prácticas', es decir, desarrollando su vida ordinaria a través del ejercicio de diferentes papeles (padre, artesano, vecino, etc.). Sin embargo, la persona es una, guarda una unidad básica y esencial más allá (o 'debajo de') esos papeles. Esa unidad se va desarrollando en una narración 'hacia' el florecimiento, 'hacia' y 'en' la vida buena. El florecimiento se refiere, por ello, a la finalidad última del individuo en cuanto persona (no en cuanto padre, artesano o vecino).

La relación entre las virtudes y el florecimiento es estrecha, esencial. El individuo se va haciendo y, en ese 'ir haciéndose', va también definiendo el bien progresivamente. Las virtudes (morales e intelectuales) son las cualidades que permiten al individuo esa definición (1991b, p. 262).

Primeramente, con la ayuda de los mayores (los padres generalmente) las virtudes permiten al joven ir distanciándose de los deseos más inmediatos para poder analizarlos y juzgar su contenido, y así orientarlos (tranformándolos o encauzándolos) hacia lo que realmente es el bien (1999a, pp. 87-89). Para MacIntyre, no hay que olvidar que el desarrollo moral tiene como punto de partida la condición animal originaria del ser humano (1999a, ix-x). Posteriormente, con la práctica personal, son también las virtudes las que capacitan al individuo para distinguir por sí mismo los bienes que están en juego en una acción y lo que puede dañarlos, y para hacer posible que esos bienes sean premisas de un razonamiento cuya conclusión ha de ser una acción buena (1999a, pp. 92-93). 
De este modo -como afirma Mauri (2018, pp. 225-230)- las virtudes devienen parte esencial de la vida buena. "Las virtudes y los vicios tienen que ver con nosotros 'qua' seres humanos, no 'qua' padres, consumidores o partícipes en la vida política" (1990d: 90). No acertar con las normas de la virtud es fracasar como seres humanos, pues las mismas virtudes son necesarias para todas las áreas de la vida (aunque exijan actuaciones distintas según el ámbito y el momento) (1990d, p. 90). Señala MacIntyre que por eso las virtudes son parte constitutiva de un fin que es un todo que incluye y requiere esa parte como un componente propio (1981, pp. 148-149). Son "internal means" (medios internos) (1981, p. 184), al modo en que las primeras jugadas de una partida de ajedrez son un medio para jugar esa partida -ejemplifica nuestro autor-. No son, pues, un medio 'externo', un medio en el sentido de una actividad distinta de un estado terminal que es causalmente eficaz para producirlo (1988, pp. 131-132).

Por eso la virtud tiene un valor intrínseco: "the acts required by virtues are each of them worth performing for their own sake" 4 (1999a, pp. 111-112). Para un individuo maduro, la misma virtud acaba siendo razón suficiente para actuar (1999a, pp. 159-160). Por esto dice MacIntyre que las virtudes deben ser practicadas sin reparar en las consecuencias, es decir, aunque no se alcance -por ejemplo- un determinado bien interno de una práctica en un determinado momento (1981, pp. 198-199). Y es que la virtud tiene un valor intrínseco que le viene dado finalmente por ser el medio (interno) para el florecimiento del ser humano: "[virtues] are constitutive parts of human flourishing"5 -afirma el autor (1999a, pp. 111-112) -. Por ello una virtud es una razón última o premisa suficiente de actuación porque el hombre y la comunidad florecen de un modo específicamente humano en el ejercicio de las virtudes (1999a, p. 113).

Hemos dicho que toda práctica tiene unas normas. El individuo debe asumirlas y seguirlas si quiere alcanzar el bien interno de esa práctica, bien que le da sentido. De igual manera, y como no toda práctica por el hecho de serlo es buena, existen unas normas del florecimiento. Estas normas son, según MacIntyre, las que componen la ley natural y su respeto permite al individuo alcanzar el florecimiento como ser humano. Así como el florecimiento es el criterio de las prácticas, la ley natural es el criterio de las normas de las prácticas. Para MacIntyre, pues, las normas tienen sentido solo como guías hacia un bien. La autonomía humana, por tanto, actúa de modo natural guiada por unas normas que ella no ha creado (1990a, pp. 133-134, 139; 1990c, p. 344; 1992, p. $143 ; 1994$, p. 173; 1996a, pp. 676-677; 1999a, pp. ix-x; 2000, p. 113; 2006, p. vii; 2009, pp. 3, 23, 24).

La virtud en el esquema macintyreano tiene sentido solo a la luz de estos conceptos explicados: práctica y florecimiento. Las virtudes, para MacIntyre, vendrían a ser esas cualidades necesarias para distinguir y realizar el bien y aplicar las normas, tanto en

4 “...las acciones requeridas por el ejercicio de las virtudes son, todas ellas, valiosas por sí mismas".

5 “...[las virtudes] son elementos constitutivos del florecimiento humano". 
el ámbito de las prácticas como en el del florecimiento. Definir el florecimiento como máximo bien y los bienes relativos a él sólo es posible con las virtudes (1981, pp. 191, 195-196; 1988, pp. 115-116; 1990a, pp. 130-131, 139; 1990b, pp. 35-36, 41-42; 1991b, p. 262; 1999a, pp. 91-92, 159-161).

Por todo ello, MacIntyre considera que la mejor manera de fundamentar y explicar la acción humana -el razonamiento práctico que hay detrás de una acción-es el bien, lo bueno para el individuo. En Whose Justice? Which Rationality? (1988, pp. 2-3), nuestro autor distingue la existencia de, al menos, tres concepciones fundamentales del razonamiento práctico.

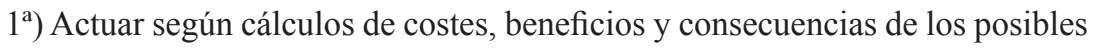
cursos de acción.

$2^{\mathrm{a}}$ ) Actuar bajo los límites que cualquier persona racional e imparcial consideraría razonables.

$3^{\mathrm{a}}$ ) Actuar según el bien último y verdadero del ser humano.

A la luz de lo dicho sobre el florecimiento, nuestro autor aboga por la tercera. El ser humano puede y debe reconocer los bienes propios de su especie -como hombre- y orienta sus acciones hacia fines en función de ellos (MacIntyre, 1999a, pp. 23-24).

La verdadera justificación última de una acción, por tanto, es que ayuda a alcanzar lo que es bueno para el individuo -para mí- qua ser humano (aunque también pueda haber razones de otra índole), es decir, lo que me hace florecer como ser humano: el florecimiento es el telos en la racionalidad práctica (MacIntyre, 1999a, pp. 112-113, 159-160). Tal es la premisa inicial del razonamiento práctico y la base de la validez práctica y teórica- de todas las virtudes, y lo que hace racional una acción para MacIntyre (MacIntyre, 1999a, pp. 159-160). Por ello, siguiendo a Aristóteles (Ética nicomáquea 1112b, pp. 33-34), opina que el razonamiento o la deliberación práctica se refiere sólo a los medios, no al fin, pues se delibera sobre fines concretos en la medida en que son medios para otros fines ulteriores -y para un fin último-(MacIntyre, 1999a, p. 106).

Desde esa perspectiva, el esquema general del razonamiento práctico que propone MacIntyre se estructura, en su esquema más básico, en tres pasos:

$\left.1^{\circ}\right)$ Premisas acerca del bien humano,

$2^{\circ}$ ) Pasos intermedios que especifican lo que exigen las virtudes para alcanzarlo,

$3^{\circ}$ ) Acción mejor y más posible aquí y ahora (MacIntyre 1999a, pp. 158-159).

El carácter de las prácticas y del florecimiento (junto con la propia falibilidad del ser humano) hace que el individuo requiera la ayuda de otros para triunfar en la consecución del bien. Efectivamente, el individuo no puede alcanzar los bienes internos de las prácticas sin desarrollar ciertas virtudes ni aprender a reconocer y a aplicar ciertas normas, para lo cual requiere la ayuda de otras personas en distintos grados y distintos momentos de toda su vida. Lo mismo ocurre con el florecimiento (1988, pp. 122-123). 


\section{Definición de 'virtud'}

A la luz de lo expuesto es posible exponer y entender la noción de virtud que propone MacIntyre. En función de los conceptos de 'florecimiento' ('unidad narrativa') y de 'práctica', MacIntyre va construyendo o acotando la definición de virtud en After Virtue y Dependent Rational Animals. En ese proceso se observa que las virtudes no solo ayudan a las prácticas sino también a la constancia en la búsqueda de lo bueno y al conocimiento de ello y del propio individuo.

La primera definición de virtud -parcial y provisional- que nuestro autor ofrece en After Virtue se refiere más a la práctica y dice así: "A virtue is an acquired human quality the possession and exercise of which tends to enable us to achieve those goods which are internal to practices and the lack of which effectively prevents us from achieving any such goods"6 (1981, p. 191). Más adelante, el autor se sirve de una definición más rica y amplia: "The virtues therefore are to be understood as those dispositions which will not only sustain practices and enable us to achieve the goods internal to practices, but which will also sustain us in the relevant kind of quest for good, by enabling us to overcome the harms, dangers, temptations and distractions which we encounter, and which will furnish us with increasing self-knowledge and increasing knowledge of good"7 (1981, p. 219).

Complementariamente, MacIntyre afirma, en Dependent Rational Animals, que las virtudes son excelencias (excellences), son "those qualities of mind and character that enable someone both to recognize the relevant goods and to use the relevant skills in achieving them"8 (1999a, pp. 91-92).

Vemos, pues, que las virtudes se manifiestan e influyen en todos los ámbitos de la vida del individuo. Si bien nacen o se dan inicialmente en las prácticas, su alcance es mucho mayor: la vida como unidad (con su 'telos') y el razonamiento práctico (1981, pp. 201-203; 1999a, p. 120). A lo largo del tratamiento de diferentes temas (práctica, florecimiento, etc.), el autor va poniendo ejemplos de distintos momentos o esferas del ser humano en los que las virtudes aparecen como necesarias: el cuidado y la educación de otros, la protección (propia y de los demás), la falta de compasión..., etc. (1999a, pp. 97-98). Pero, finalmente, viene a decir, en síntesis, que las virtudes influyen en la actitud o reacción del individuo en distintas situaciones, en las relaciones sociales y

6 'Una virtud es una cualidad humana adquirida, cuya posesión y ejercicio tiende a hacernos capaces de lograr aquellos bienes que son internos a las prácticas y cuya carencia nos impide efectivamente el lograr cualquiera de tales bienes".

7 "[Las virtudes son] aquellas disposiciones que no sólo mantienen las prácticas y nos permiten alcanzar los bienes internos a las prácticas, sino que nos sostendrán también en el tipo pertinente de búsqueda de lo bueno, ayudándonos a vencer los riesgos, peligros, tentaciones y distracciones que encontremos y procurándonos creciente autoconocimiento y creciente conocimiento del bien".

8 “...las cualidades intelectuales y de carácter que permiten a una persona identificar los bienes pertinentes y emplear las habilidades necesarias para conseguirlos...”. 
en el razonamiento práctico de todo agente (1999a, pp. 92, 98, 119-120), es decir, en todos los ámbitos de la vida.

Ya hemos visto que las virtudes son necesarias para distinguir, valorar y realizar los bienes internos a las prácticas (1981, pp. 198-199) y que ayudan a entender y a vivir el florecimiento humano (1991b, p. 262; 1999a, p. 112). Al abarcar todos los ámbitos de la vida del individuo, pues, las virtudes influyen en todas las acciones -todos los razonamientos prácticos- que el agente realiza.

Se ha comentado que las reglas (o normas) son enunciados que ponen límites a una actuación guiándola (hacia el bien). Las reglas son guías generales del comportamiento. Esa generalidad y esa limitación provocan que, a la hora de actuar, ninguna norma pueda determinar por sí misma cómo responder correctamente a una situación. Ello se debe a que las reglas que hay que respetar siempre-las del florecimiento-, como "No matar", nunca bastan, según nuestro autor, para determinar cómo hay que comportarse en la acción concreta, pues definen solo parcialmente el tipo de bondad al que se aspira (el todo que es el florecimiento) (1990a, p. 139; 1992, p. 143; 1999a, p. ix-x). Y, en el caso de las normas que dependen de las circunstancias -las de las prácticas principalmente-, es necesario juzgar su pertinencia o no (1988, pp. 31-32).

El uso y la aplicación concretos de unas reglas no dependen ni están especificados por otras reglas sino que dependen de las cualidades del sujeto (virtudes), de ahí que la excelencia nunca se pueda reducir a mera aplicación de reglas $(1988$, p. 32). Por tanto, no existe ningún tipo de norma, ni prohibiciones inviolables ni prescripciones que puedan orientar, mover y concretar la acción por sí mismas (como único elemento, sin contar con dichas cualidades) (1999a, p. 94; 1998, p. 106).

Las premisas acerca del bien humano, parte primera del razonamiento práctico que precede a una acción, contienen unas normas cuya aplicación depende de esos "pasos intermedios" en que las exigencias de las virtudes se concretan (segunda parte) para concluir en una acción. Las virtudes, pues, 'operativizan', 'materializan' las reglas. Para nuestro autor -siguiendo a Tomás de Aquino-, las reglas requieren las virtudes porque actuar según unas reglas sólo es bueno si es expresión de virtudes y porque el seguimiento de las reglas en sí mismo requiere la virtud. Comportarse virtuosamente, pues, implica más que el mero uso de normas (1999a, pp. 88-89, 93-94).

\section{Las virtudes y el papel de la prudencia}

Una vez explicado el concepto de virtud y su importancia, conviene dedicar una breve explicación a enumerar sucintamente las virtudes que son más relevantes en el pensamiento de nuestro autor y, así, poder entender el papel que juega la prudencia.

En general, MacIntyre considera que tanto las virtudes morales como las intelectuales son importantes para el desarrollo moral del individuo (1991b, p. 262). Entre ellas, hay unas virtudes más básicas o destacables que otras. Tales virtudes son fundamentales para el individuo, para los demás y para la relación interpersonal. 
Pueden variar en contenido concreto pero siempre han sido reconocidas como virtudes -y virtudes importantes- en toda sociedad (1981, pp. 192-193; 1966, p. 77), pues sus mínimas exigencias no son sino requerimientos de la ley natural (1994, pp. 184185). A la luz de lo expresado por nuestro autor en distintos momentos y obras de su trayectoria, se puede afirmar que esas virtudes esenciales para él son estas: la justicia, el valor (o valentía, fortaleza), la veracidad (u honestidad), la templanza, la integridad, la constancia, la paciencia, la consideración de los otros y la generosidad ${ }^{9}$ (1966, p. 78; 1981, pp. 192-193; 1994, pp. 184-185; 1999a, pp. 119-120).

Sobre la templanza, nuestro autor afirma que ayuda de una manera fundamental a controlar y encauzar ciertas tendencias. Gracias a ella, la persona sabe orientar su atracción a algunos bienes inferiores para seguir, avanzar y triunfar en la definición y consecución práctica del bien superior último. Sin la templanza, el sujeto considerará independientes esos bienes inferiores -su búsqueda- con respecto al bien supremo; considerará las reglas iniciales que le guiaban hacia ese bien supremo como opuestas a esos bienes inmediatos; $\mathrm{y}$, finalmente, desaparecerá la idea de un bien que puede ser esencialmente superior a otros (MacIntyre 1992, pp. 145).

Aunque las virtudes mencionadas son importantes, tanto en el nivel teórico (definición del bien y distinción de bienes particulares) como en el práctico (aplicación/ realización efectiva de un bien particular partiendo del general), MacIntyre destaca especialmente el papel de una virtud: la prudencia, la 'phronesis'. Un primer signo del papel eminente que nuestro autor da a esta virtud es que, en la mayoría de los casos en que la menciona o la aborda, le da un tratamiento aparte y distinto del que da a otras virtudes.

MacIntyre afirma que la prudencia es la virtud central de la vida práctica para Aristóteles y Tomás de Aquino (1990b, pp. 41-42), dos autores referenciales para él (especialmente a partir de los años 80-90). De igual modo, él también le da un papel preeminente, como se ve en obras como Whose Justice?, Firts Principles... o Dependent Rational Animals (MacIntyre 1981, pp. 191, 195-196; 1988, pp. 115-116; 1990a, pp. 130-131, 139; 1990b, pp. 35-36, 41-42; 1991b, p. 262; 1999a, pp. 91-92, 159-161).

En Whose Justice?, MacIntyre (1988: 115-116) define la 'phronesis' como “the exercise of a capacity to apply truths about what it is good for such and such a type of person or for persons as such to do generally and in certain types of situation to oneself on particular occasions. The 'phronimos' is able to judge both which truths are relevant

9 Conviene destacar que, en Dependent Rational Animals, MacIntyre (1999a, pp. 119-120) sintetiza la justicia y la generosidad en la virtud de la 'justa generosidad', que consiste en una mezcla o una relación complementaria y dependiente entre la justicia y la generosidad, y que el autor presenta como la virtud más adecuada para unas relaciones armónicas entre los individuos. 
to him in his particular situation and from that judgment and from his perception of the relevant aspects of himself and his situation to act rightly"10.

En Three Rival Versions of Moral Enquiry (1990a, p. 139), la define como "the virtue of being able in particular situations to bring to bear the relevant universals and to act so that the universal is embodied in the particular" $"$.

Por tanto, en primera instancia, la prudencia, tal como la define MacIntyre, presenta -a nuestro juicio- tres características esenciales. La primera es que busca conectar lo general (principios generales) con lo particular, es decir, con una situación que exige determinados deberes partiendo de dichos principios. La segunda es que, para ello, es necesario que el agente sepa detectar las características esenciales o relevantes de esa situación particular. La tercera es que su finalidad última es una acción, es decir, no se dirige a la especulación teórica (aunque la supone y por eso busca 'encarnarla', 'particularizarla') sino a un acto concreto ejecutable, a la práctica.

Se ha mencionado el concepto de 'situación' particular. En After Virtue, hablando sobre la inteligibilidad de la acción de un individuo, MacIntyre afirma que ambos conceptos ('inteligibilidad' y 'acción') se comprenden y se conectan en la 'situación', en 'una' situación ('situation'). La noción de inteligibilidad es el lazo vinculante de la noción de acción con la noción de historia (MacIntyre 1981, p. 214), vínculo que se da material y concretamente en la situación. Según nuestro autor, lo fundamental de la situación es que tiene una historia a la luz de la cual se entiende, una historia que, si bien es individual, está compuesta también de las historias de los otros (1981, pp. 207-208; 1999a, pp. 148-149). La situación está formada por esas historias (personal e interpersonal), por el hecho concreto que realiza el agente, por su intención y por unas circunstancias (MacIntyre 1981, pp. 206-207). Es la prudencia -podemos concluir- la que da al individuo la cualidad para saber gestionar y resolver dicha situación.

Por el proceso complejo que entraña la prudencia y por la misma complejidad de una situación, la prudencia es una virtud dependiente, no autosuficiente: requiere las virtudes morales para su formación, crecimiento y práctica. Para MacIntyre, como para Aristóteles y Tomás de Aquino, la relación entre las virtudes morales y la 'phronesis' es de mutua necesidad. De hecho, sostiene que toda virtud moral "cannot be exercised without 'phronesis' also being exercised"12 (1988, pp. 115-116).

El motivo que aduce es que, sin las virtudes morales, los deseos no pueden estar informados por la razón, no pueden transformarse ni ser eficaces en cuanto deseos para

10 "...el ejercicio de la capacidad de aplicar verdades acerca del bien para tal o cual tipo de persona o para personas en general y en ciertos tipos de situaciones a uno mismo en ocasiones particulares. El 'phronimos' es capaz de juzgar no sólo qué verdades son relevantes para él en su situación particular sino que también, a partir de ese juicio y de su percepción de los aspectos relevantes de sí mismo y de su situación, cómo actuar rectamente".

11 "...la virtud consistente en ser capaz de remitirse, en las situaciones particulares, a los universales pertinentes y actuar de modo que el universal se encarne en el particular".

12 "...no puede ejercerse sin que se ejerza también la "phronesis". 
lo que la razón prescriba. "It is the virtues which enable the desire which is 'prohairesis' to be rational desire" 13 -afirma en Whose Justice? - (1988, pp. 136-137). Gracias a ellas -leemos en Three Rival Versions- la 'orexis', deseo sentido, deviene 'prohairesis', un deseo guiado por la razón (1990a, pp. 64-65).

Al hablar de las virtudes en general, se ha comentado que son necesarias para distinguir, valorar y realizar los bienes internos de las prácticas y que ayudan a entender y a vivir el florecimiento humano. Se ha comentado también que las normas (sean las que informan las prácticas, sean las que informan el florecimiento) son guías generales del comportamiento; y que el uso y la aplicación concretos de unas reglas no dependen ni están especificados por otras reglas sino que dependen de las cualidades del sujeto (virtudes). A la luz de lo comentado en párrafos anteriores, es posible ver que, para nuestro autor, en todos esos procesos, la prudencia tiene un papel central. Gracias a ella, el individuo sabe qué reglas aplicar a una situación y de qué manera aplicarlas, de modo que el bien -lo mejor-se encarne en tal situación.

Vemos, pues, que es la virtud de la prudencia (y las virtudes que implica) la que posibilita el carácter necesariamente causal (causal de acciones concretas) del razonamiento práctico. Sin ella, el razonamiento práctico sería incorrecto como razonamiento e ineficaz como práctico $^{14}$. $\mathrm{Y}$ es precisamente ese carácter causal lo que define a la ética como saber, como saber que no habla tanto de lo que es sino de lo que debe ser, punto en el que radica su dificultad como disciplina de conocimiento (MacIntyre 1966, pp. 49-50). Así pues, se puede concluir que para actuar racionalmente se requieren las virtudes y, en concreto, la virtud de la prudencia (MacIntyre 1999, pp. 158-159). Como afirma Loria (2018, p. 277), para nuestro autor, la racionalidad -su ejercicio, su desarrollo- está estrechamente relacionada con las cualidades morales.

Como se ha comentado ya, la prudencia permite al individuo aplicar correctamente las reglas (a una situación concreta). Pero MacIntyre señala que, no obstante, el calado de esa virtud es mucho más hondo, pues no solo influye en la aplicación de normas sino también en su definición. Es decir, la prudencia es necesaria para definir y/o establecer normas morales. Aunque la prudencia tiene un papel eminente en ello (y quizá por esa razón), tanto las virtudes morales como las intelectuales son necesarias para una correcta definición de las normas (MacIntyre 1988, pp. 115-116). Son las virtudes las que deben guiar la definición de las reglas, no al revés, como hace la moral moderna (MacIntyre 1981, pp. 119-120).

Ello no implica para nuestro autor que todas las normas dependan o 'emanen' del ser humano (de su racionalidad, de su voluntad, de sus virtudes, de su prudencia). Las normas que componen la ley natural (que, como hemos visto, son el criterio del florecimiento humano) tienen una existencia y una autoridad independientes de la

13 "Son las virtudes las que capacitan al deseo, que es la 'prohairesis', para ser un deseo racional".

14 MacIntyre no formula esta idea explícitamente, aunque puede derivarse de su esquema descrito. 
voluntad del individuo. Por eso afirma MacIntyre, en "How Can We Learn What Veritatis Splendor Has To Teach?", que el marco de aplicación de las reglas es el definido por los preceptos de la ley natural (especialmente los negativos) y, en consecuencia "The acquisition of that virtue [phronesis] is impossible without a recognition of the rational authority of the precepts of the natural law, most of all perhaps of the negative exceptionless precepts" 15 . Por ello, tales preceptos capacitan a la racionalidad y a la autonomía humanas, no la merman (MacIntyre 1994, p. 183).

Para acabar, conviene comentar un último ámbito en el que MacIntyre da una importancia especial a la prudencia: el del conocimiento. Aunque nuestro autor relaciona la prudencia con el razonamiento práctico - con las acciones-, señala, asimismo, que esta virtud influye también en el conocimiento como actividad, es decir, en la práctica de la investigación racional o científica y, en concreto, en la filosofía moral. La virtud de la prudencia, al ser una virtud de naturaleza moral e intelectual, influye en el conocimiento que el ser humano puede adquirir sobre el hombre y el mundo.

Esto ocurre de modo más patente en el estudio de textos específicamente de moral. Nuestro autor sostiene que, para que textos como la Ética nicomáquea o la Summa sean significativos e inteligibles, la persona ya debe tener ciertas cualidades o virtudes -en grado mínimo, pero presente y real- que son precisamente las que recibirán justificación en esa lectura. Esos textos responden a unas preguntas y de un modo, y el lector, para aprovecharlos, debe formular esas preguntas y de ese modo (MacIntyre 1992, pp. 148-149).

Otro ejemplo de ello aparece en Three Rival Versions, cuando MacIntyre, hablando sobre cómo leer la Summa Theologiae, explica que, para aprender por qué se requieren las virtudes y la obediencia a la ley moral -si la persona quiere realizar o lograr su bienya son necesarias, hasta cierto grado y de alguna manera, ciertas virtudes. Entre esas ciertas virtudes, señala, en primer lugar, las virtudes intelectuales; y, dentro de ellas, destaca la prudencia, la virtud de la inteligencia y del juicio prácticos. No obstante, ésta sólo se puede poseer si se poseen las virtudes morales previamente -como se ha dicho-(MacIntyre 1990a, pp. 130-131)."It is not after all paradoxical to assert that we must already have those virtues at least to some degree if we are to understand why we need to have them"16 (MacIntyre 1992, p. 151). Como afirma Mas (1996, p. 168), la virtud es, a la vez, base y producto de la investigación racional.

MacIntyre comparte la tradición clásica (Sócrates, Platón, Tomás de Aquino, etc.) en su concepción de la relación entre la virtud y el conocimiento: son conceptos o

15 "La adquisición de esa virtud [phronesis] es imposible sin reconocer la autoridad racional de los preceptos de la ley natural, sobre todo de esos preceptos negativos que no admiten excepción".

16 “...no es paradójico afirmar que debemos tener esas virtudes, al menos en algún grado, si hemos de comprender por qué necesitamos tenerlas". 
realidades inseparables, se requieren mutuamente ${ }^{17}$. Según nuestro autor, toda forma de investigación filosófica es práctica en sus implicaciones, y toda investigación práctica es filosófica en sus presupuestos (MacIntyre 1990a, pp. 128-129). Si la 'phronesis' une lo particular y lo general y es esencial para establecer y definir correctamente lo general -lo universal-, uniendo así lo teórico y lo práctico (MacIntyre 1988, pp. 115116; 1990a, p. 139), es lógico que MacIntyre considere que la carencia de esta virtud tenga implicaciones notables para la investigación racional, pues podría impedir llegar a la definición del 'telos' de una investigación o de una ciencia (MacIntyre 1990b, pp. 41-42; 1998, pp. 104-106).

Hemos visto que, sin virtudes morales, los deseos no pueden estar informados por la razón ni transformarse ni ser eficaces como deseos para lo que la razón prescribe (MacIntyre 1988, pp. 137-138), de modo que la adquisición de virtudes determina en gran medida lo que resulta agradable y útil a una persona (MacIntyre 1999, pp. 87-88). También hemos visto que las virtudes (morales e intelectuales) son necesarias para la definición y la aplicación de las reglas, la prudencia especialmente (MacIntyre 1988, pp. 115-116).

Se puede deducir de estas ideas que el verdadero avance intelectual se consigue cuando los deseos son rectos y las reglas propias de la disciplina estudiada se aplican y se saben aplicar según tales deseos, lo cual se consigue a través de las virtudes. Es lógico concluir esto cuando MacIntyre sostiene que son las virtudes las cualidades que hacen fructíferos, honestos y progresistas los debates y la argumentación dentro de una comunidad y de una tradición; y que son ellas las que mantienen la vida y el dinamismo de una tradición como cuerpo de diálogo y transmisor de conocimiento (MacIntyre 1981, pp. 223-224). Ver las cosas tal como son (hechos) implica ya un elemento moral, cierta disposición moral del sujeto (valores) ante la realidad (MacIntyre 1983, p. 13).

\section{Conclusión}

A modo de conclusión, se puede afirmar que, en el pensamiento de MacIntyre, la prudencia (la 'phronesis') juega un papel fundamental, central, como en el de Aristóteles

17 Sócrates (y Platón y Aristóteles) afirmaba la necesidad de ciertas virtudes y disposiciones para poder iniciar una buena investigación filosófica. Por eso, Nietzsche aconsejaba no 'entrar' en la dialéctica socrática para evitar previsibles o posibles conclusiones. Platón, en la misma línea, sostenía que el investigador debía llegar a ser un determinado 'tipo de persona'. Tal visión es -afirma MacIntyre- opuesta al enciclopedismo, que habla de la 'verdad' sin perspectiva ni consideración del factor humano personal. Detrás de dicha visión (la de Sócrates hasta Tomás de Aquino), está la concepción de la filosofía como un arte, una 'techné', que tiene, debe tener unas determinadas características cuando está en buenas condiciones. Otro ejemplo de esta postura para MacIntyre es San Agustín, en cuyo pensamiento el conocimiento, la virtud y la autoridad son conceptos inseparables, como denota su epistemología (MacIntyre 1990a, pp. 60-61, 84-85). 
y Tomás de Aquino. Ciertamente, MacIntyre no desarrolla de manera sistemática o extensa la definición y el contenido de la prudencia, pero, a la luz de lo comentado, se puede constatar que es una virtud que vertebra su concepción general de las virtudes y de la vida del ser humano (en los niveles de las prácticas y del florecimiento).

En resumen, podemos decir que la importancia de la virtud de la prudencia radica para MacIntyre en tres puntos.

1) Capacita al individuo para aplicar a una situación concreta y particular principios generales, de modo que le permite distinguir las circunstancias relevantes según el bien general buscado.

2) Una acción correcta concreta no es producto de aplicar reglas, aunque las requiera y las contenga: la aplicación (y la definición) de reglas no depende de una regla, sino de la prudencia.

3) De ahí que la carencia de esta virtud tenga implicaciones importantes para la investigación racional: sin ella, no es posible para el individuo avanzar en el conocimiento intelectual y, en concreto, en la investigación moral.

\section{Referencias bibliográficas}

Corral, C. (1997), “Acción, identidad y tradición: el argumento narrativo”, en Mauri, M.; Román, B. y otros (1997), Crisis de valores. Modernidad y tradición (Un profundo estudio de la obra de A. MacIntyre). Barcelona: EditEuro; pp. 115142.

MacIntyre, A. (1966), A Short History of Ethics, Macmillan, N. York; y (1967), Routledge and Kegan P., London, $2^{\mathrm{a}}$ edic. (1998), Routledge and Kegan Paul, London. En este trabajo, las referencias de página se indican según la $2^{\mathrm{a}}$ edición (1998) aunque, por claridad y orden, se indique el año de la $1^{\mathrm{a}}$ edición.

(1981), After Virtue: A Study in Moral Theory, Duckworth, London. $2^{\mathrm{a}}$ edición: (1984) After Virtue: A Study in Moral Theory. N. D. Press, Notre Dame (Indiana). En este trabajo, las referencias de página se indican según la $2^{\mathrm{a}}$ edición (1984) aunque se indique el año de la $1^{\mathrm{a}}$ edición.

(1983), Revisions: Changing Perspectives in Moral Philosophy, Alasdair MacIntyre y Stanley Hauerwas (eds.), University of Notre Dame Press, Notre Dame (Indiana)/London. MacIntyre escribe: "Preface" (p. vii), "Moral Philosophy: What Next?”, pp. 1-15. 498-513.

(1984), "Does Applied Ethics rest on a Mistake?", The Monist, v. 67:

(1988), Whose Justice? Which Rationality?, University of Notre Dame Press, Notre Dame (Indiana).

(1990a), Three Rival Versions of Moral Enquiry: Encyclopaedia, Genealogy, and Tradition. London: Duckwort. 
(1990b), First Principles, Final Ends and Contemporary Philosophical Issues. Reproducido en 1998, The MacIntyre Reader, K. Knight, ed.; pp. 171201.

(1990c), "The Privatization of Good. An Inaugural Lecture", The Review of Politics, v. 52 (n. ${ }^{\circ}$ 3, Summer): 344-361.

(1990d), "Después de Tras la virtud" (entrevista de R. Yepes a MacIntyre), Atlántida, vol. 1 (n. ${ }^{\circ}$ 4): 87-95.

(1991a), "Community, Law, and the Idiom and Rhetoric of Rights", Listening: Journal of Religion and Culture, v. 26, (n. 2): 96-110.

(1991b), "Nietzsche O Aristotele?", en Conversazioni Americane, Giovanna Borradori, (ed.), Editori Laterza, Roma-Bari, pp. 169-187. Reimpreso con el título “An Interview with Giovanna Borradori” en 1998, The MacIntyre Reader, Kelvin Knight (ed.), Polity Press, Cambridge; pp. 255-66. Usamos esta reimpresión para este trabajo aunque indicamos en el texto el año de la publicación original.

(1992), "Plain Persons and Moral Philosophy: Rules, Virtues and Goods", American Catholic Philosophical Quarterly, v. 66, (n. 1, Winter): 3-20. Reimpreso en 1998, The MacIntyre Reader, Kelvin Knight, ed.; pp. 13652. La página se toma de la edición en este libro (indicando el año original).

(1994), "How Can We Learn What Veritatis Splendor Has To Teach?", The Thomist (v. 58): 171-195.

(1996a), "Wahre Selbsterkenntnis durch Verstehen unserer selbst aus der Perspektive anderer" (interview with Dmitri Nikulin), Deutsche Zeitschrift für Philosophie, v. 44 (n. ${ }^{\circ}$ 4): 671-683.

(1996b), "Natural Law As Subversive: The Case of Aquinas", Journal of Medieval and Early Modern Studies, v. 26 (n. ${ }^{\circ}$ 1, winter): 61-83.

(1998), “Aquinas's Critique of Education: Against His Own Age, Against Ours", en Philosophers on Education: New Historical Perspectives, A. Oksenberg Rorty, ed. London-N. York: Routledge; pp. 95-108.

(1999a), Dependent Rational Animals: Why Human Beings Need the Virtues, Duckworth, London; y Open Court Publishing, Chicago and La Salle (Illinois).

(1999b), "Social Structures and their Threats to Moral Agency," Philosophy (74): 311-329.

(2000), "Theories of Natural Law in the Culture of Advanced Modernity", in Common Truths: New Perspectives on Natural Law, Edward B. McLean, ed., Wilmington, Delaware: ISI Books; pp. 91-115.

(2006), The Tasks of Philosophy. Selected essays, vol. 1, N. York: CUP.

(2009), "Intractable Moral Disagreements" y "From Answer to Questions: A Response to the Responses", en Cunningham, L. ed., Intractable Disputes about the Natural Law. Alasdair MacIntyre and Critics, University of Notre Dame Press, Indiana, pp. 1-52 (cap. 1) y pp. 313-351 (cap. 10). 
(2016), Ethics in the Conflicts of Modernity. Cambridge: CUP.

Loria, M. (2018), "A ação racional e o bem humano", en Carvalho, H. ed., Tradição, Moralidade e Racionalidade: Em diálogo com Alasdair MacIntyre. Porto Alegre, Editora Fi y EDUFPI; pp. 273-310.

Mas, S. (1996), "El tema de la virtud: A. MacIntyre, lector de Aristóteles", Revista de Filosofia (9/15): 159-181.

Mauri, M. (1999), Liberalisme i llibertat (“Alasdair MacIntyre: la tradició de les virtuts"), Qüestions de vida cristiana, n. 194, Pub. Abadia de Montserrat; pp. 45-56.

(2018) "Conhecimento, vida e virtude moral", en Carvalho, H., ed., Tradição, Moralidade e Racionalidade: Em diálogo com Alasdair MacIntyre. Porto Alegre: Editora Fi y EDUFPI; pp. 225-244.

Miller, D. (1994), "Virtues, Practices and Justice", en Horton, J. y Mendus, S., eds., After MacIntyre: Critical Perspectives on the Work of Alasdair MacIntyre, Cambridge: Polity Press. 